\title{
Efeitos transgeracionais do tabagismo materno durante a gestação e amamentação
}

\author{
Transgerational effects of maternal tobacco in pregnancy and \\ breastfeeding
}

Recebido em: 08/02/2017

Aceito em: 18/03/2018
Miyoko MASSAGO; Elaine Schultz DWORAK

Universidade Estadual de Maringá, Av. Colombo, 5790, Zona 7,

CEP87020-900. Maringá, PR, Brasil.E-mail:mi_massago@hotmail.com

\section{ABSTRACT}

Approximately 250 million women are smokers worldwide, even in pregnancy, and it can cause serious consequences to their childrens. Therefore, the present study aimed to perform a literature review on the transgenerational effects of maternal smoking during pregnancy, using Lilacs, Pubmed and Scielo as the database. It was possible to observe that maternal smoking during pregnancy can lead to physiological, biological alterations and feeding habits in the different stages of the individual's development, causing irreversible damage to her/him due to cardiometabolic schedules during the crucial stages of their development.

Keywords: children; smoking; pregnancy

\section{RESUMO}

Mundialmente, aproximadamente 250 milhões de mulheres são fumantes, inclusive na gravidez podendo ocasionar graves consequências às suas proles. Diante disso, o presente trabalho objetivou-se realizar uma revisão de literatura sobre os efeitos transgeracionais do tabagismo materno durante a gestação e amamentação, usando como banco de dados o Lilacs, Pubmed e Scielo. Foi possível observar que o tabagismo materno durante a gestação pode levar a alterações fisiológicas, biológicas e hábitos alimentares nas diferentes fases do desenvolvimento do indivíduo ocasionando danos irreversíveis ao mesmo devido às programações cardiometabólicas durante as etapas cruciais do seu desenvolvimento.

PALAVRAS-CHAVE: crianças; tabagismo; gravidez

\section{INTRODUÇÃO}

O tabagismo, ato de consumir produtos que contenham tabaco, é a principal causa de mortes por doenças crônicas não transmissíveis, podendo desencadear quase 50 doenças fatais e incapacitantes (1). Sendo assim, é considerado um grave problema de saúde pública. No entanto, mundialmente mais de 1,2 bilhões de indivíduos são fumantes (2), destes 250 milhões são mulheres (3).
O consumo do tabaco durante a gestação e a amamentação ocasiona graves consequências à saúde materna e fetal, levando a altos gastos médicos relacionados às complicações fetais, como o baixo peso ao nascer (4), e consequentemente um aumento na assistência em Unidade de Terapia Intensiva (UTI) neonatal (5), além de desencadear algumas situações patológicas e/ou tendências durante as diferentes etapas da vida $(6,7)$, como obesidade infantil (8) e preferências alimentares na vida adulta (9).

Por outro lado, a cessação do tabagismo no início da gestação acarreta no aumento da saúde fetal (10) e 
a abstinência após o parto evita a exposição passiva do neonato às toxinas do tabaco, diminuindo o risco de infecções respiratórias (11). No entanto, apesar de 25 a $40 \%$ das fumantes pararem de fumar ou reduzirem o consumo de cigarros quando tomam ciência de que estão grávidas (12-14), aproximadamente 12 a 15\% apresentam recaídas durante a gestação (15).

Segundo o Ministério da Saúde, entre os anos 1980 e $2007,15,2 \%$ dos fumantes receberam algum tipo de aconselhamento pelos profissionais de saúde e 6,7\% utilizaram alguma farmacoterapia auxiliar na cessação do hábito de fumar (16), reforçando a importância da assistência farmacêutica na cessação do tabagismo. A literatura relata que o farmacêutico pode desencorajar o fumo, educar sobre os malefícios do tabagismo, enfatizar os riscos à saúde das pessoas, encaminhar ao Programa de Cessação do Tabagismo e oferecer assistência àqueles que estão em tratamento (17), promovendo assim o aumento do abandono do vício. Baseado nestas informações, no presente trabalho foi realizada uma revisão da literatura sobre o tabagismo materno durante a gestação e a amamentação e os efeitos transgeracionais, quanto às consequências ocasionadas por este vício.

\section{MÉTODOS}

Durante os anos 2017 e 2018, foi realizada uma pesquisa bibliográfica em banco de dados Lilacs (um), Pubmed (trinta e cinco) e Scielo (seis), utilizando as palavras-chaves, "tabagismo materno", "gestação", em artigos nos idiomas inglês e português e publicados entre os anos 1972 e 2015.

Em seguida os artigos referentes ao assunto foram selecionados de acordo com o grau de relevância. Neste contexto, foram analisados os problemas que o consumo de tabaco na gestação pode ocasionar à saúde das futuras gerações, desde os prejuízos fetais até a vida adulta.

\section{RESULTADOS E DISCUSSÃO}

Efeitos no feto. Durante o estágio fetal, o tabagismo materno pode ocasionar problemas como placenta prévia, episódios de hemorragia materna, abortos espontâneos, nascimentos prematuros, mortes fetais e aceleração do batimento cardíaco (18), pois a nicotina leva à vasoconstricção das artérias placentárias, diminuindo a concentração do oxigênio e o fluxo sanguíneo na mãe, diminuindo a sua oferta para o feto, e levando à menor absorção de nutrientes pelo mesmo $(19,20)$.

O tabagismo durante a gravidez está relacionado também à síndrome de morte súbita do bebê, provavelmente pela baixa capacidade da medula adrenal do feto responder reflexivamente à hipóxia, não havendo a liberação de catecolaminas para a redistribuição do fluxo sanguíneo para o cérebro e o coração, não ocorrendo, assim, a manutenção da frequência cardíaca (11).

O consumo de tabaco ocasiona baixo ganho de peso nos fetos no segundo e no terceiro trimestres de gestação, resultante dos efeitos tóxicos da nicotina, cianeto, monóxido de carbono e outras substâncias tóxicas (21). $\mathrm{O}$ comprometimento no ganho de peso ocorre devido à hipóxia fetal, à vasoconstricção das veias e das artérias da placenta e à supressão dos hormônios do crescimento placentário e da função de fatores endócrinos de crescimento $(22,23)$.

A carboxihemoglobina, originada da ligação da hemoglobina com o monóxido de carbono produzido durante a combustão do tabaco, provoca a hipóxia tecidual, estimulando a eritropoiese e a elevação do hematócrito fetal implicando em hiperviscosidade sanguínea, aumento do risco de infarto cerebral no neonato e mau desempenho da placenta (24-26). No sistema nervoso do feto, o gás carbônico pode causar lesões neurológicas temporárias e/ou permanentes (27) e no sistema cardiovascular ocorre a elevação da frequência cardíaca e hipertrofia miocárdica (28).

O comprometimento do sistema imunológico provocado pelo tabagismo materno aumenta o risco de abortamento (29), devido à ruptura prematura das membranas, secundárias às infecções locais facilitadas pela toxicidade do fumo (11) e pela redução da concentração do ácido ascórbico (vitamina C) no líquido amniótico (30). A vitamina $\mathrm{C}$ é fundamental na defesa imunológica do organismo (31) e na formação do colágeno da membrana aminiocoriônica (32). Além disso, há menor transporte de aminoácidos pela placenta das mães fumantes, interferindo na baixa síntese proteica e contribuindo para o mau desenvolvimento da membrana aminiocoriônica $(33,34)$.

Outro fator responsável pelo aumento do abortamento em fumantes é a redução da síntese do óxido nítrico, relaxante do miométrio, e inativação do fator de ativação das plaquetas, podendo ocasionar contrações uterinas e parto prematuro (35).

Efeitos nos neonates. O consumo do tabaco durante a gestação pode causar alterações no desenvolvimento 
fetal (23); consequentemente, os filhos de mães fumantes apresentam maiores chances de nascer com baixo peso (22) além de afetar outros parâmetros como o comprimento da circunferência occipital-frontal $(21,22)$.

O consumo de um a cinco cigarros reduz o peso de neonatos em 111 gramas; de seis a dez cigarros, em 175 gramas; e mais de 10 cigarros, em 236 gramas (36). Nos neonatos, o tabagismo materno durante a fase gestacional, provoca também síndrome de abstinência do tabagismo fetal, que é caracterizada por choros e cólicas (37).

No lactante, o tabaco apresenta efeitos tóxicos como diarreia, vômitos, taquicardia, sonolência e choque, pois a nicotina presente no leite materno é rapidamente absorvida pelo intestino do lactante podendo se acumular nos tecidos nos neonatos $(38,39)$. Além disso, as mulheres que fumam são menos propensas a amamentar os seus filhos e quando amamentam, a duração é menor do que em não fumantes, uma vez que a nicotina tem um efeito negativo no suprimento do leite materno devido à supressão dos níveis de prolactina, inibindo assim a sua produção e, consequentemente, os lactantes ganham peso mais lentamente (40).

A nicotina, nos lactantes, leva a alterações nos padrões de sono e vigília, danos ao fígado e aos pulmões, redução das células $\beta$-pancreáticas, redução da oferta de iodo pelo leite materno e dos níveis de superóxido dismutase e catalase, elevação da concentração sérica de malondialdeído, e à hiperleptinemia (41).

Ademais, é possível observar a presença de 2,9 vezes mais nicotina no leite materno comparado à quantidade encontrada no plasma das mulheres em período de lactação (42) e aproximadamente dez vezes mais nicotina na urina dos lactantes amamentados por fumantes em relação ao observado naqueles alimentados artificialmente (43).

Efeitos na infância e adolescência. Os filhos de mães fumantes apresentam menor crescimento em estatura, quando comparados aos filhos de não fumantes (44), comprometimento pulmonar $(45,46)$, maior risco de desenvolverem obesidade, aterosclerose e diabetes $(8,47)$ e alterações da cognição $(48)$, e comprometimento no desenvolvimento psicomotor (49) e sexual no jovem (50).

A exposição fetal aos compostos do tabaco compromete o crescimento dos pulmões e leva à redução das pequenas vias aéreas, implicando em alterações funcionais respiratórias na infância, como a asma durante os sete primeiros anos da vida que persistem ao longo da vida $(46,47,51,52)$.

O desenvolvimento da obesidade em crianças de mães que fumaram durante a gravidez está associado ao hipotireoidismo (53). A correlação entre excesso de peso na infância e na adolescência e o consumo do cigarro é maior no primeiro trimestre da gestação (54), pois a interrupção do vício pode levar ao estresse ou ansiedade materno, elevando os níveis de cortisol e influenciando o metabolismo dos filhos (55).

O consumo excessivo de lipídios na adolescência de indivíduos expostos à nicotina na vida intrauterina pode estar associado à diminuição da amígdala (região envolvida no processamento da recompensa) (56), enquanto que o diabetes é ocasionado pela perda de células $\beta$-pancreáticas e diminuição da tolerância à lactose (57).

As alterações da cognição e o comprometimento do desenvolvimento psicomotor e sexual no jovem estão associados aos efeitos secundários a neurotoxicidade da nicotina, a qual interage de forma inadequada com os receptores nicotínicos colinérgicos, prejudicando a neurogênese e a sinaptogênese (58).

Efeitos na vida adulta. Indivíduos adultos expostos ao tabagismo materno durante a gestação preferem carboidratos às proteínas (9) e estão associados ao aumento da desordem de conduta, déficit de atenção, hiperatividade e delinquência juvenil nos homens e de delitos na vida adulta (59).

\section{CONCLUSÃO}

O tabagismo materno durante a gravidez e a amamentação pode afetar todas as fases da vida da prole, podendo ocasionar graves consequências,pois toda a vida do indivíduo pode sofrer programação cardiometabólica durante as etapas cruciais do seu desenvolvimento. Portanto, há a necessidade de se pesquisar novas medidas para promover aumento na cessação tabácica $\mathrm{e}$ na assistência farmacêutica aos fumantes, para melhorar a qualidade de vida não só dos fumantes, mas também dos seus descendentes. 


\section{REFERÊNCIAS}

1. BRASIL. Ministério da Saúde. Instituto Nacional do Câncer. Tabagismo: um grave problema de saúde pública. 2007. [Cited 2017 December 06]. Available from: http://www1.inca.gov.br/inca/Arquivos/t_Tabagismo.pdf

2. OMS.OrganizaçãoMundialdaSaúde.Tobacco.FactsSheet 339. 2017. [Cited 2017 November 11]. Available from: http://www.who.int/mediacentre/factsheets/fs339/en/

3. OMS. Organização Mundial da Saúde. 10 facts on gender and tobacco. 2010. [Cited 2017 November 11]. Available from: http://www.who.int/gender/documents/ 10facts_gender_tobacco_en.pdf.

4. Halpern R, Schaefer ES, Pereira AS, Arnt EM, Bezerra JP, Pinto S. Risk factors for low birth weight in a rural community in southern Brazil. J Pediatr 1996;72:369-373.

5. Adams EK, Miller VP, Ernst C, Nishimura BK, Melvin C, Merritt R. Neonatal health care costs related to smoking during pregnancy. Health Econ 2002;11:193-206. DOI: $10.1002 /$ hec.660.

6. Parlee SD, MacDougald OA. Maternal nutrition and risk of obesity in offspring: the Trojan horse of developmental plasticity. Biochim Biophys Acta, 2014;1842(3): 495506. DOI: 10.1016/j.bbadis.2013.07.007.

7. Bedzhov I, Graham SJ, Leung CY, Zernicka-Goetz M. Developmental plasticity, cell fate specification and morphogenesis in the early mouse embryo. Philos Trans R Soc Lond B Biol Sci, 2014;369(1657): 20130538. DOI: 10.1098/rstb.2013.0538.

8. Nagel G, Arnold FJ, Wilhelm M, Link B, Zoellner I, Koenig W. Environmental tobacco smoke and cardiometabolic risk in young children: results from a survey in south-west Germany. Eur Heart J, 2009;30(15): 18851893. DOI: 10.1093/eurheartj/ehp180.

9. Ayres C. O tabagismo materno durante a gestação e o consumo alimentar na vida adulta. [Dissertação]. Porto Alegre: Faculdade de Medicina, Universidade Federal do Rio Grande do Sul, 2014. 56p.

10. Butler NR, Goldstein H, Ross EM. Cigarette smoking in pregnancy: its influence on birth weight and perinatal mortality. Br Med J. 1972;:127-130. DOI: 10.1136/ bmj.2.5806.127.

11. Leopércio W, Gigliotti A. Tabagismo e suas peculiaridades durante a gestação: uma revisão crítica. J Bras Pneumol 2004;30(2):176-185. DOI: 10.1590/S180637132004000200016.

12. Windsor RA, Lowe JB, Perkins LL, Smith-Yoder D, Artz L, Crawford M, Amnburgy K, Boyd NR Jr. Health education for pregnant smokers: its behavioral impact and cost benefit. Am J Public Health 1993;83:201-206.

13. Valbo A, Schioldborg P. Smoking in pregnancy: a follow-up study of women unwilling to quit. Addict Behav 1993;18:253-237. DOI: 10.1136/bmjopen-2017-018746.
14. Floyd RL, Rimer BK, Giovino GA, Mullen PD, Sullivan SE. A review of smoking in pregnancy: effects on pregnancy outcomes and cessation efforts. Annu Rev Public Health 1993;14:379-411. DOI: 10.1146/annurev. pu.14.050193.002115

15. Petersen L, Handel J, Kotch J, Podedwomy T, Rosen A. Smoking reduction during pregnancy by a program of self-help and clinical support. Obstet Gynecol 1992; 79:924-30.

16. BRASIL. Ministério da Saúde. Instituto Nacional do Câncer.. Pesquisa Especial de Tabagismo - PETab: relatório Brasil. 2008. [Cited 2018 February 2]. Available from: http://bvsms.saude.gov.br/bvs/publicacoes/pesquisa_especial_tabagismo_petab.pdf

17. Silva EV. O papel do farmacêutico comunitário na cessação do tabagismo. Bol Farmacoterap 2007;3: 1-5.

18. Maia JA, Pereira LA, Menezes F. A. Consequências do uso de drogas durante a gravidez. Rev Enf Contemp, 2015;4(2):121-128. DOI: 2317-3378rec.v4i2.664.

19. Vielwerth S, Jensen R, Larsen T, Greisen G. The impact of maternal smoking on fetal and infant growth. Early Hum Dev, 2007;83(8):491-495. DOI: 10.1016/j.earlhumdev.2006.09.010.

20. Fasting MH, Oien T, Storro O, Nilsen T-IL, Johnsen R, Vik T. Maternal smoking cessation in early pregnancy and offspring weight status at four years of age. A prospective birth cohort study. Early Hum Dev. 2009;85(1):19-24. DOI: 10.1016/j.earlhumdev.2008.05.009.

21. Bosley A, Sibert J, Newcombe R. Effects of maternal smoking on fetal growth and nutrition. Arch Dis Child. 1981;56(9): 727-729.

22. Zhang L, González-Chica DA, Cesar JA, Mendoza-Sassi RA, Beskow B, Larentis N, Blosfeld T. Tabagismo materno durante a gestação e medidas antropométricas do recém-nascido: um estudo de base populacional no extremo sul do Brasil. Cad Saude Publica, 2011;27(9): 1768-1776. DOI: 10.1590/S0102-311X2011000900010

23. Prabhu N, Smith N, Campbell D, Craig LC, Seaton A, Helms PJ, Devereux G, Turner SW. First trimester maternal tobacco smoking habits and fetal growth. Thorax, 2010;65(3):235-240. DOI: 10.1136/thx.2009.123232.

24. Gabriel R, Alsat E, Evain-Brion D. Alteration of epidermal growth factor receptor in placental membranes of smokers: relationship with intrauterine growth retardation. Am J Obstet Gynecol 1994;170:1238-1243. DOI: 10.1016/S0002-9378(13)90441-5.

25. Buchan PC. Cigarette smoking in pregnancy and fetal hyperviscosity. Br Med J 1983;1286:1315. DOI: 10.1136/bmj.286.6374.1315

26. Bureau MA et al. Maternal cigarette smoking and fetal oxygen transport: a study of P50, 2,3-Diphosphoglycera- 
te, total hemoglobin, hematocrit, and type of hemoglobin in fetal blood. Pediatrics 1983;72:22-26.

27. Penney DG. Effects of carbon monoxide exposure on developing animals and humans. In: Penney DG, editor. Carbon monoxide. Boca Raton: CRC Press; 1996. p.109144.

28. Clubb FJ Jr, Penney DG, Baylerian MS, Bishop SP. Cardiomegaly due to myocyte hyperplasia in perinatal rats exposed to $200 \mathrm{ppm}$ carbon monoxide. J Mol Cell Cardiol 1986;18:477-486. DOI: 10.1016/S0022-2828(86)809130 .

29. Meyer MB, Tonascia JA. Maternal smoking, pregnancy complications, and perinatal mortality. Am J Obstet Gynecol 1977; 128:494-502. DOI: 10.1016/00029378(77)90031-X.

30. Barrett B, Gunter E, Jenkins J, Wang M. Ascorbic acid concentration in amniotic fluid in late pregnancy. Biol Neonate 1991;60:333-5. DOI: 10.1159/000243426.

31. Anderson R. Effects of ascorbate on leucocytes: part II. Effects of ascorbic acid and calcium and sodium ascorbate on neutrophil phagocytosis and post-phagocytic metabolic activity. South Afr Med J 1979;56:401-404.

32. Phillips CL, Tajima S, Pinnell SR. Ascobic acid and transforming growth factor-B-1 increase collagen biosynthesis via different mechanismis: coordinate regulation of pro-alpha-1(I) and pro-alpha-1(III) collagens. Arch Biochem Biophys 1982;295:397-403.

33. Sastry BV. Placental toxicology: tobacco smoke, abused drugs, multiple chemical interactions, and placental function. Reprod Fertil Dev 1991;3:355-372.

34. French JI, Mcgregor JA. The pathobiology of premature rupture of membranes. Semin Perinatol 1996;20:344368. DOI: 10.1016/S0146-0005(96)80002-4.

35. Keelan JA, Coleman M, Mitchell MD. The molecular mechanisms of term and preterm labor: recent progress and clinical implications. Clin Obstet Gynecol 1997;40:460-478.

36. Bernstein IM, Mongeon JA, Badger GJ, Solomon L, Heil SH, Higgins ST. Maternal smoking and its association with birth weight. Obstet Gynecol 2005;106(5Pt1):986991. DOI: 10.1097/01.AOG.0000182580.78402.d2.

37. Matheson I, Rivrud GN. The effect of smoking on lactation and infantile colic apud Galão AO, Macedo BR, Ribeiro RVP, Ribeiro RVP, Vanin CMM. Comparação entre puérperas fumantes e ex-fumantes com relação ao tempo de amamentação e suas consequências sobre a saúde dos recém-nascidos. Rev Bras Cardiol, 2011;57(3):379-385.

38. Eckerdt NdS, Corradi-Webster CM. Sentidos sobre o hábito de fumar para mulheres participantes de grupo de tabagistas. Rev Lat Am Enferm. 2010;18(spe):641-647. DOI: 10.1590/S0104-11692010000700022.

39. Luck W, Nau H. Nicotine and cotinine concentrations in serum and milk of nursing smokers. Br J Clin Pharmacol 1984;18:9-15.
40. Mello PRB, Pinto GR, Botelho C. The influence of smoking on fertility, pregnancy and lactation. J Pediatr 2001;77(4):257-264. DOI: 10.1590/S002175572001000400006

41. Primo CC, Ruela P, Brotto L, Garcia TR, Lima E. Efeitos da nicotina materna na criança em amamentação. Rev Paulista Pediatr, 2013;31(3):392-397. DOI: 10.1590/ S0103-05822013000300018

42. Amir LH. Maternal smoking and reduced duration of breastfeeding: a review of possible mechanisms apud Primo CC, Ruela PBF, Brotto LDA, Garcia TR, Lima EF. Efeitos da nicotina maternal na criança em amamentação. Rev Paulista Pediatr, 2013;31(3):392-397. DOI: 10.1590/S0103-05822013000300018.

43. Mascola MA, Vunakis HV, Tager IB, Speizer FE, Hanrahan JP. Exposure of Young infants to environmental tobacco smoke: breast-feeding among smoking mothers. Am J Public Health, 1998;(88): 893-896.

44. Martínez-Mesa J, Menezes AM, González DA, Horta BL, Matijasevich A, Gigante DP. Life course association of maternal smoking during pregnancy and offspring's height: data from the 1993 Pelotas (Brazil) birth cohort. J Adolesc Health, 2012;51(Suppl 6): S53-S57.

45. Gilliland FD, Berhane K, McConnell R, Gauderman WJ, Vora H, Rappaport EB, Avol E, Peters JM. Maternal smoking during pregnancy, environmental tobacco smoke exposure and childhood lung function. Thorax 2000;55:271-276. DOI: 10.1136/thorax.55.4.271.

46. Gilliland FD, Li YF, Peters JM. Effects of maternal smoking during pregnancy and environmental tobacco smoke on asthma and wheezing in children. Am J Respir Crit Care Med 2001;163:429-436. DOI: 10.1164/ajrccm.163.2.2006009.

47. Lisboa PC, Oliveira E, Moura EG. Obesity and endocrine dysfunction programmed by maternal smoking in pregnancy and lactation. Front Physiol 2012;3:437. DOI: 10.3389/fphys.2012.00437.

48. Cutler AR, Wilkerson AE, Gingras JL, Levin GD. Prenatal cocaine and/or nicotine exposure in rats: preliminary findings on long-term cognitive outcome and genital development at birth. Neurotoxicol Teratol 1996;18:635643. DOI: 10.1016/S0892-0362(96)00125-0.

49. Schlumpf M, Gahwiler M, Ribary U, Lichtensteiger W. A new device for monitoring early motor development: prenatal nicotine-induced changes. Pharmacol Biochem Behav 1988;30:199-203. DOI: 10.1016/00913057(88)90444-3.

50. Peters DA, Tang S. Sex-dependent biological changes following prenatal nicotine exposure in the rat. Pharmacol Biochem Behav 1982;17:1077-1082. DOI: 10.1016/0091-3057(82)90497-X.

51. Jaakkola JJ, Gissler M. Maternal smoking in pregnancy, fetal development, and childhood asthma. Am J Public Health, 2004;94(1):136-140. 
52. Gonzalez-Barcala F-J, et al. Impact of parental smoking on childhood asthma. J Pediatr 2013;89(3):294-299. DOI: 10.1016/j.jped.2012.11.001.

53. Oliveira E, Moura E, Santos-Silva A, Fagundes A, Rios A, Abreu-Villaca Y, Nogueira Neto JF, Passos MC, Lisboa PC. Short-and long-term effects of maternal nicotine exposure during lactation on body adiposity, lipid profile, and thyroid function of rat offspring apud Primo CC, Ruela PBF, Brotto LDA, Garcia TR, Lima EF. Efeitos da nicotina materna na criança em amamentação. Rev Paul Pediatr, 2013;31(3):392-397. DOI: 10.1590/S010305822013000300018.

54. Muraro AP, Gonçalves-Silva RMV, Ferreira MG, Sichieri R. Effect of the exposure to maternal smoking during pregnancy and childhood on the body mass index until adolescence. Rev Saude Publica, 2015;49. DOI: 10.1590/ S0034-8910.2015049005423.

55. Sarkar P, Bergman K, O'Connor T, Glover V. Maternal antenatal anxiety and amniotic fluid cortisol and testos- terone: possible implications for foetal programming. J Neuroendocrinol, 2008;20(4):489-496. DOI: 10.1111/ j.1365-2826.2008.01659.

56. Haghighi A, et al. Prenatal exposure to maternal cigarette smoking, amygdala volume, and fat intake in adolescence. JAMA psychiatry, 2013;70(1): 98-105. D.O.I. 10.1001/archgenpsychiatry.2012.1101.

57. Bruin JE, Kellenberger LD, Gerstein HC, Morrison KM, Holloway AC. Fetal and neonatal nicotine exposure and postnatal glucose homeostasis: identifying critical windows of exposure. J Endocrinol, 2007;194(1):171-178. DOI: 10.1677/JOE-07-0050.

58. Slotkin TA. Fetal nicotine or cocaine exposure: which one is worse? J Pharmacol Exp Ther 1998;285:931-945.

59. Räsänen, P, Hakko H, Isohanni M, Hodgins S, Järvelin M-R, Tiihonen J. Maternal smoking during pregnancy and risk of criminal behavior among adult male offspring in the Northern Finland 1966 Birth Cohort. Am J Psychiatry, 1999;156(6): 857-862. DOI: 10.1176/ajp.156.6.857. 